Editorial

\title{
Are beta oscillations always anti-kinetic in Parkinson's disease?
}

\author{
Francesco Asci ${ }^{\mathrm{a}}$, Alexandre Eusebio ${ }^{\mathrm{b}, \mathrm{c}}$, Antonio Suppa ${ }^{\mathrm{a}, \mathrm{d}, *}$ \\ a IRCCS Neuromed Institute, Pozzilli, IS, Italy \\ b Institut de Neurosciences de la Timone, UMR7289, CNRS and Aix-Marseille Univ, Marseille, France \\ ${ }^{\mathrm{c}}$ APHM, Aix-Marseille Univ, Department of Neurology and Movement Disorders, CHU Timone, Marseille, France \\ ${ }^{\mathrm{d}}$ Department of Human Neurosciences, Sapienza University of Rome, Italy
}

\section{Introduction}

Parkinson's Disease (PD) is a neurodegenerative disorder clinically characterized by the prominent association of motor signs, including bradykinesia, rigidity and resting tremor (Armstrong and Okun, 2020). The pathophysiology of parkinsonian motor signs and symptoms has been deeply investigated in the last three decades. Experimental studies in non-human primate models of PD, as well as invasive neuronal recordings in PD patients undergoing deep brain stimulation (DBS), have shown abnormal oscillations in the beta band in specific basal ganglia structures, such as the subthalamic nucleus, and cortical motor areas, such as the primary motor cortex (Brown, 2007; Hahn and McIntyre, 2010; Holgado et al., 2010; Iskhakova et al., 2021). This evidence has led to the dominant hypothesis that neurodegeneration of nigrostriatal dopaminergic projections promotes abnormal beta oscillations in the basal ganglia-thalamo-cortical motor loop (Jenkins et al., 1992; Nachev et al., 2008). Accordingly, current consensus attributes to beta rhythms in the basal ganglia-thalamo-cortical motor loop the role of an anti-kinetic oscillation responsible for the main motor symptoms in PD (Brown, 2007; Iskhakova et al., 2021). However, experimental studies in healthy primates and humans have demonstrated that beta oscillations also mediate relevant physiological functions, for example, when specific networks are activated during visually-guided or corrected and goal-directed voluntary movements (Androulidakis et al., 2006; Chakrabarti et al., 2014; Jahani et al., 2020; Stetson and Andersen, 2014). The dorsal visual stream is indeed an integrated cortical network including specific fronto-parietal regions, involved in several aspects of visuo-motor integration, such as reaching in response to object motion or self-motion (Fogassi and Luppino, 2005). So far, the possible pathophysiologic role of beta-rhythms in the dorsal visual network has been poorly investigated in patients with PD during the execution of visually-guided and goal-directed voluntary movements.

\footnotetext{
* Corresponding author at: Department of Human Neurosciences, Sapienza University of Rome and, IRCCS Neuromed Institute, Viale dell'Università 30, 00185 Rome, Italy.

E-mail address: antonio.suppa@uniroma1.it (A. Suppa).
}

The present volume of Clinical Neurophysiology includes the study by Sparks et al. (2022), who used electrocorticographic recordings to investigate functional connectivity between critical nodes of the dorsal visual network (i.e., posterior parietal cortex - PPC, and dorsal lateral premotor cortex - LPMC), in a cohort of 13 PD patients undergoing DBS. The authors have compared the PPC-to-LMPC functional connectivity in response to internally cued (IC) and visual externally cued (EC) actions. It is known that PD patients manifest greater abnormalities in motor planning and execution in response to IC than visual EC actions. This likely reflects at least two main pathophysiological mechanisms: 1) prominent impairment of medial cortical areas, which are mostly responsible for IC movements (Chung et al., 2018) and; 2) partially spared activation of lateral networks, including the dorsal visual network, which are known to assist visual EC movements (Galletti and Fattori, 2018). Sparks et al. (2022) compared neuronal recordings from PPC and LMPC, in patients with PD undergoing IC and EC motor tasks consisting of directional movements of a joystick-controlled by the right or left hand toward the direction of arrows on a screen. In both IC and EC trials, as main kinematic variable, the authors measured the reaction time (RT) from the visual cue to hand movement onset. As neurophysiological measure, the authors included the absolute peak of the power spectrum in the beta frequency in PPC and LPMC. Also, to assess the phase coupling and direction of information flow between PPC and LPMC, the authors considered two additional measures: the debiased weighted phase lag index (dwPLI) and the Granger causality (GC). Although the absolute power in the beta band decreased in PPC and LMPC, both the PPC-LPMC dwPLI and GC increased in the beta band and more prominently during EC than IC trials. Still, then authors found significant correlations between kinematic (i.e., RT) and neurophysiological (i.e., dwPLI) measures, during EC (i.e., the greater the PPC-LPMC dwPLI, the shorter the RT). Hence, the study by Sparks et al. (2022) suggests that the increased PPC-toLPMC functional connectivity in the beta band reflects a compensatory activation of the dorsal visual network able to improve motor execution in PD patients (Rubinstein et al., 2002). On a more general note, the study of Sparks et al. raises doubts about the common assumption that beta rhythms are always anti-kinetic in PD. 
When considering the neurophysiological observations of Sparks et al. several possible limitations should be taken into account. For instance, the study includes a rather small cohort of PD patients and lacks a control group. A second point concerns the experimental design since also the IC trials were triggered by an external cue. Lastly, although non-demented, PD patients may manifest subclinical visuo-spatial abnormalities owing to the subtle degeneration of the dorsal visual network (Aarsland et al., 2021; Armstrong, 2015). The study by Sparks et al. (2022) however, is relevant to the debate on beta oscillations in PD. Moreover, the study opens new opportunities for innovative non-pharmacological strategies in patients with PD. A possible perspective would be to improve motor execution in PD by enhancing functional coupling in the beta band between critical nodes of the dorsal visual network through non-invasive brain stimulation. To this purpose, transcranial alternating current stimulation (tACS) has been proved to reliably modulate cortical rhythms by entraining endogenous oscillations (Guerra et al., 2021, 2020). The next steps towards the understanding of the causal role of beta rhythms in the dorsal visual stream could therefore be to examine whether beta tACS applied over specific nodes of the dorsal visual network can improve motor execution in PD patients.

\section{Funding}

None.

\section{Acknowledgements}

None.

\section{References}

Aarsland D, Batzu L, Halliday GM, Geurtsen GJ, Ballard C, Ray Chaudhuri K, Weintraub D. Parkinson disease-associated cognitive impairment. Nat Rev Dis Primers 2021;7(1). https://doi.org/10.1038/s41572-021-00280-3.

Androulidakis AG, Doyle LMF, Gilbertson TP, Brown P. Corrective movements in response to displacements in visual feedback are more effective during periods of 13-35 Hz oscillatory synchrony in the human corticospinal system. Eur J Neurosci 2006;24:3299-304.

Armstrong MJ, Okun MS. Diagnosis and treatment of parkinson disease: a review. JAMA 2020;323(6):548. https://doi.org/10.1001/iama.2019.22360.
Armstrong RA. Oculo-visual dysfunction in Parkinson's disease. J Parkinsons Dis 2015:5(4):715-26.

Brown P. Abnormal oscillatory synchronisation in the motor system leads to impaired movement. Curr Opin Neurobiol 2007;17(6):656-64.

Chakrabarti S, Martinez-Vazquez P, Gail A. Synchronization patterns suggest different functional organization in parietal reach region and dorsal premotor cortex. J Neurophysiol 2014;112(12):3138-53.

Chung JW, Burciu RG, Ofori E, Coombes SA, Christou EA, Okun MS, Hess CW Vaillancourt DE. Beta-band oscillations in the supplementary motor cortex are modulated by levodopa and associated with functional activity in the basal ganglia. Neuroimage Clin 2018;19:559-71.

Fogassi L, Luppino G. Motor functions of the parietal lobe. Curr Opin Neurobiol 2005;15(6):626-31.

Galletti C, Fattori P. The dorsal visual stream revisited: Stable circuits or dynamic pathways? Cortex 2018;98:203-17.

Guerra A, Asci F, D’Onofrio V, Sveva V, Bologna M, Fabbrini G, Berardelli A, Suppa A. Enhancing gamma oscillations restores primary motor cortex plasticity in Parkinson's disease. J Neurosci 2020;40(24):4788-96.

Guerra A, Colella D, Giangrosso M, Cannavacciuolo A, Paparella G, Fabbrini G, et al. Driving motor cortex oscillations modulates bradykinesia in Parkinson's disease. Brain 2021 Jul 10; awab257. doi: 10.1093/brain/awab257. Online ahead of print.

Hahn PJ, McIntyre CC. Modeling shifts in the rate and pattern of subthalamopallidal network activity during deep brain stimulation. J Comput Neurosci 2010;28 (3):425-41.

Holgado AJN, Terry JR, Bogacz R. Conditions for the generation of beta oscillations in the subthalamic nucleus-globus pallidus network. J Neurosci 2010;30 (37):12340-52.

Iskhakova L, Rappel P, Deffains M, Fonar G, Marmor O, Paz R, Israel Z, Eitan R, Bergman $\mathrm{H}$. Modulation of dopamine tone induces frequency shifts in corticobasal ganglia beta oscillations. Nat Commun 2021;12(1). https://doi.org/ 10.1038/s41467-021-27375-5.

Jahani A, Schwey A, Bernier P-M, Malfait N. Spatially distinct beta-band activities reflect implicit sensorimotor adaptation and explicit re-aiming strategy. J Neurosci 2020;40(12):2498-509.

Jenkins IH, Fernandez W, Playford ED, Lees AJ, Frackowiak RSJ, Passingham RE Brooks DJ. Impaired activation of the supplementary motor area in Parkinson's disease is reversed when akinesia is treated with apomorphine. Ann Neurol 1992;32(6):749-57.

Nachev P, Kennard C, Husain M. Functional role of the supplementary and presupplementary motor areas. Nat Rev Neurosci 2008;9(11):856-69.

Rubinstein TC, Giladi N, Hausdorff JM. The power of cueing to circumvent dopamine deficits: a review of physical therapy treatment of gait disturbances in Parkinson's disease. Mov Disord 2002;17(6):1148-60.

Sparks H, Cross KA, Choi JW, Courellis H, Thum J, Koenig E, Pouratian N. Dorsal visual stream is preferentially engaged during externally guided action selection in Parkinson Disease. Clinical Neurophysiology 2021. https://doi.org 10.1016/i.clinph.2021.11.077.

Stetson C, Andersen RA. The parietal reach region selectively anti-synchronizes with dorsal premotor cortex during planning. J Neurosci 2014;34(36):11948-58. 